\title{
COMMENTARY
}

\section{Lymphotoxin-Beta Receptor Signaling Is Crucial for the Vascularization of Transplanted Metanephros}

\author{
Valerio Brizi* and Christodoulos Xinaris ${ }^{*}$
}

From the Istituto di Ricerche Farmacologiche Mario Negri IRCCS, * Centro Anna Maria Astori, Science and Technology Park Kilometro Rosso, Bergamo, Italy; and the University of Nicosia Medical School, ${ }^{\dagger}$ Nicosia, Cyprus

\section{Growing Embryonic Kidneys in Living Animals}

Although approximately 18,000 patients with end-stage kidney disease receive a donor organ each year in the USA, ${ }^{1}$ there are more than 5 times as many patients on waiting lists. The shortage of donor organs has caused the waiting period to increase to over three years and the mortality rate by $5 \%$ to $10 \%$. Growing metanephroi (fetal kidney primordia) in living animals that could be used for replacement therapies may help bridge this gap.,3

Over the last two decades, studies have shown that it is possible to grow new kidneys in adult living hosts or to integrate new filtering nephrons into neonatal kidneys. Embryonic kidney rudiments implanted beneath the renal capsule, ${ }^{4,5}$ within the omentum ${ }^{5,6}$ or into tunnels in the cortices of host kidneys ${ }^{7}$ become integrated into living animals and exhibit key renal functions, such glomerular filtration and macromolecule reabsorption. Cultured fetal kidneys $^{8}$ or organoids made from embryonic kidney cells ${ }^{9}$ also become vascularized and form mature and functional glomeruli following in vivo transplantation. More recent studies have shown that mouse ${ }^{10}$ and human ${ }^{11}$ kidney primordia transplanted into the lymph node (LN) can also grow and mature into functional miniorgans.

Growing embryonic anlagens in situ has important technical and clinical benefits/advantages. The developing kidney is less immunogenic than the adult kidney because it contains fewer antigen-presenting cells and expresses fewer MHC class I and class II antigens. ${ }^{2}$ Compared to embryonic stem cells or induced pluripotent stem cells (PSCs), metanephric cells are already committed to a genetic program of renal development, obviating the need to preprogram cell fate and engineer a complete organ de novo. Nevertheless, there are also important challenges that must be addressed before these approaches achieve clinical relevance. First, unnatural ectopic environments do not allow fetal kidneys to develop into fully structurally and functionally mature organs (embryonic grafts reach approximately $4 \%$ of natural kidney size). Second, the ability of transplanted fetal kidneys to grow differentially according to the environment, results in a great variation in the outcomes of transplantation (in terms of efficient vascularization, and structural and functional maturation). Surprisingly, fetal organs grow differentially, even in the same animal, when transplanted into different sites. ${ }^{12}$ Identifying the cellular and molecular mechanisms that coordinate ectopic organ development would significantly improve the efficacy of the existing methods and hopefully produce kidney tissues that can be used for replacement therapies.

One important step toward understanding the mechanisms that rule ectopic organogenesis has been taken by the studies conducted by Francipane et al ${ }^{13}$ which are published in the current issue of The American Journal of $\mathrm{Pa}$ thology. By transplanting kidney rudiments into either the LNs of mice undergoing lymphotoxin-beta receptor (LT $\beta R$ ) antagonist treatment or the omenta of $\mathrm{Lt}_{\mathrm{Br}} \mathrm{r}^{--}$mice,

Supported by a European Foundation for the Study of Diabetes/Novo Nordisk Programme for Diabetes Research in Europe, the Associazione per la Ricerca sul Diabete Italia, and Bellco S.p.A.

Accepted for publication October 1, 2019.

Disclosures: None declared.

Address correspondence to Christodoulos Xinaris, Ph.D., Department of Molecular Medicine, Istituto di Ricerche Farmacologiche Mario Negri IRCCS, Centro Anna Maria Astori, Science and Technology Park Kilometro Rosso, Via Stezzano, 8724126 Bergamo, Italy. E-mail: christodoulos.xinaris@marionegri.it 
Francipane et al demonstrate that host LT $\beta R$ signals are crucial for the development of a well-vascularized kidney graft, as well as its survival and growth. The absence of LT $\beta$ R signals also impairs compensatory glomerular growth in response to renal mass reduction, without affecting normal kidney development.

This commentary discusses the significance of these findings as well as points out the current challenges in utilizing these findings in regenerative nephrology. It further highlights the avenues that should be explored next.

\section{Vascularization of Glomeruli Engrafted into Mouse LN Might Be Driven by the Host LT $\beta R /$ NIK Axis}

Earlier studies by Francipane showed that embryonic kidneys transplanted into mouse LNs develop mature nephrons with a vascular network that originates from both host and graft-derived vascular cells. ${ }^{10,11,14}$ Although these studies demonstrated that the LN provides an appropriate niche for transplanting and growing embryonic kidneys, the cellular and molecular mechanisms that govern organogenesis within the $\mathrm{LN}$ remain unknown.

In this new study, Francipane et al investigated the molecular signals that support kidney vascularization in host lymphoid sites and omenta. Based on the observation that most glomerular endothelial cells $\left(\mathrm{CD} 34^{+}\right)$express nuclear factor $\mathrm{kB}$-inducing kinase (NIK) - a downstream target of LT $\beta R$ that is a key regulator of angiogenesis ${ }^{15}$ and LN development ${ }^{16}$ - the authors speculated that the host LT $\beta \mathrm{R} /$ NIK axis could play a role in ectopic kidney organogenesis. Francipane et al tested this hypothesis using two independent strategies: Two days before metanephros transplantation into the LNs, recipient mice were treated with a recombinant LT $\beta R-F c$ fusion protein that antagonizes LT $\beta$ R-mediated effects by engaging soluble and cell surface forms of LT $\beta R$ ligands, and transplanted kidney rudiments into the omenta of LT $\beta$ R knockout $\left(L t \beta r^{-1-}\right)$ mice. Both strategies showed that defective host LT $\beta R$ signaling is associated with decreased expression of endothelial and angiogenic markers in kidney grafts, indicating that host LT $\beta R$ signals are important for the vascularization of ectopically grown embryonic kidneys. Surprisingly, the defective host LT $\beta R$ signaling impaired glomerular vascularization in engrafted kidneys, despite the fact that the main cell source of glomerular endothelium was of graft origin.

LT $\beta R$ was not only decisive for vascularization but also for the growth of renal structures. Compared with their control counterparts, metanephroi engrafted into the $L t \beta r^{-1-}$ omentum exhibited structural alterations overtime, with complete loss of glomeruli and tubules after six weeks. Interestingly, similar results were obtained when newborn kidneys were transplanted (instead of embryonic ones), indicating that host LT $\beta$ R signals are important for the survival and growth of the developing tissue, even when the graft is extensively vascularized before transplantation.

These observations may lead to the assumption that LT $\beta$ R plays an important role in kidney organogenesis, and consequently transplanted kidneys cannot grow normally in the presence of defective LT $\beta$ R signaling. However, this was not the case: LT $\beta R$ was not essential for kidney development during embryogenesis. $L t \beta r^{-1-}$ mice exhibited normal kidney structure and vasculature, suggesting that LT $\beta R$ signaling is essential only for ectopic but not for normal organogenesis. To explain this paradox, Francipane et al assumed that LT $\beta$ R signaling is important for angiogenesis only and not for vasculogenesis, which is the main mode of vascularization during normal kidney development. However, this assumption can only partially explain the observed phenomena, because the glomerular endothelium derived almost exclusively from the donor and was indeed affected by the defective host LT $\beta$ R signaling. Still, the lymphoid microenvironment may affect the graft's vasculogenesis indirectly: it can provide the signaling cues to induce host angiogenic responses that are necessary for the survival and growth of the graft early after transplantation, and then the graft's intrinsic vascularization machinery (through secretion vasculogenic/angiogenic molecules and differentiation of endogenous endothelial progenitors) may take over to self-sustain its vascularization. In the absence of functional LT $\beta$ R signaling, both angiogenesis and vasculogenesis are affected.

Another interesting aspect of the study that requires further investigation is the contribution of LN stroma to ectopic organogenesis. Francipane et al showed that engrafted glomeruli are embedded in a host LT $\beta$ R-reactive extracellular matrix (ECM) secreted by LN stromal cells. Although LN stromal cells constitute only $1 \%$ to $5 \%$ of the LN tissue, ${ }^{17}$ they have a number of crucial functions, including the secretion of ECM components and chemokines that support hematopoietic cell maturation, ${ }^{18}$ and the regulation of vascular endothelial growth factor levels for endothelial cell proliferation. ${ }^{19}$ Therefore, identifying which type(s) of LN stromal cells support kidney vasculogenesis/ angiogenesis, as well as the stromal cell-derived ECM proteins and chemokines that are involved in these processes, would be very useful for kidney tissue engineering. This information may help improve existing methods for the differentiation of PSCs into glomerular endothelial cells, and design matrices that may enhance kidney organoid vascularization and maturation.

After observing that the number of glomerular endothelial cells that express NIK (a key regulator of neovascularization $)^{15}$ decreased in the absence of functional LT $\beta R$, the authors prudently speculated that the LT $\beta$ R/NIK axis mediated the angiogenetic signals that are required for ectopic kidney organogenesis. However, the transplantation of kidney rudiments into the omenta of $\mathrm{Nik}^{-/-}$mice revealed that NIK is dispensable for ectopic kidney vascular integration and maturation. This finding suggests either the existence of 
different targets downstream of LT $\beta$ R that lead to angiogenesis or the activation of compensatory angiogenic pathways in the absence of NIK. Another crucial issue that remains to be addressed is how the defective LT $\beta R$ signaling is associated with glomerular and tubular loss in late-stage grafts.

\section{LT $\beta R$ Signaling Is Important for Glomerular Adaptive Responses to Uninephrectomy}

The above data also raise an interesting question of clinical relevance: does LT $\beta$ R signaling play similar trophic and/or pro-angiogenetic roles in the adult kidney? To answer this question the authors uninephrectomized $L t \beta r^{-1-}$ mice and studied the remnant kidney after 10 days. Although the kidneys from wild-type and $L t \beta r^{-1-}$ mice had similar weights before surgery, the remnant kidneys of $L t \beta r^{-1-}$ mice were of a lower weight. The contralateral kidneys in wild-type mice exhibited greater glomerular enlargement and contained more erythrocytes than $L t \beta r^{-1-}$-engrafted organs. Differences in adaptive responses appeared to be confined to the glomerulus, as tubular epithelial cells from both groups exhibited a similar signal pattern of the proliferation marker Ki-67. Future studies are needed to assess whether the role of LT $\beta R$ in compensatory glomerular growth is specific to mass reduction or a general response to impaired renal function, and how LT $\beta R$ blockers $^{20}$ (used against renal or non-renal inflammations) may affect this response.

\section{Conclusions}

As novel and challenging as these findings are, they also raise important new questions. Which LT $\beta$ R-downstream pathway (or pathways) controls vascularization in ectopic kidney organogenesis? What is the precise mechanism of LT $\beta \mathrm{R}$ signaling-mediated glomerular and tubular loss in late-stage grafts?

Although the remaining questions do not allow for straightforward conclusions regarding the cellular/molecular mechanisms that support kidney organogenesis in lymphoid sites, Francipane et al's studies certainly reveal the important role that the lymphoid stromal microenvironment plays in supporting angiogenesis in ectopically transplanted embryonic kidneys. A better understanding of the properties of the LN microenvironment might have implications for growing functional organs from fetal kidney rudiments or stem-cell-derived organoids. The lack of technologies that can replicate sufficiently the organogenesis niche, prevent the realistic recapitulation of in vivo organ sizes and structures, tissue patterning and vascularization, intercellular communication, and functions. ${ }^{21}$ More in depth knowledge of the cellular/molecular mechanisms that drive ectopic kidney organogenesis will be crucial for developing protocols that will allow for better spatial and temporal control of vascularization, tissue differentiation, and maturation.

\section{Acknowledgments}

The authors thank Kerstin Mierke for the excellent editing work on the manuscript; and Rubina Novelli for constructive comments, critical reading, and corrections to the text.

\section{References}

1. Centers for Disease Control and Prevention: National Chronic Kidney Disease Fact Sheet: General Information and National Estimates on Chronic Kidney Disease in the United States. 2010. Atlanta, US Department of Health and Human Services, Centers for Disease Control and Prevention, 2010

2. Hammerman MR: Organogenesis of kidneys following transplantation of renal progenitor cells. Transpl Immunol 2004, 12:229-239

3. Xinaris C, Yokoo T: Reforming the kidney starting from a single-cell suspension. Nephron Exp Nephrol 2014, 126:107-112

4. Dekel B, Burakova T, Arditti FD, Reich-Zeliger S, Milstein O, AvielRonen S, Rechavi G, Friedman N, Kaminski N, Passwell JH, Reisner Y: Human and porcine early kidney precursors as a new source for transplantation. Nat Med 2003, 9:53-60

5. Rogers SA, Lowell JA, Hammerman NA, Hammerman MR: Transplantation of developing metanephroi into adult rats. Kidney Int 1998 , $54: 27-37$

6. Matsumoto K, Yokoo T, Matsunari H, Iwai S, Yokote S, Teratani T, Gheisari Y, Tsuji O, Okano H, Utsunomiya Y, Hosoya T, Okano HJ Nagashima H, Kobayashi E: Xenotransplanted embryonic kidney provides a niche for endogenous mesenchymal stem cell differentiation into erythropoietin-producing tissue. Stem Cells 2012, 30: $1228-1235$

7. Woolf AS, Hornbruch A, Fine LG: Integration of new embryonic nephrons into the kidney. Am J Kidney Dis 1991, 17:611-614

8. Robert B, St John PL, Hyink DP, Abrahamson DR: Evidence that embryonic kidney cells expressing flk-1 are intrinsic, vasculogenic angioblasts. Am J Physiol 1996, 271:F744-F753

9. Xinaris C, Benedetti V, Rizzo P, Abbate M, Corna D, Azzollini N, Conti S, Unbekandt M, Davies JA, Morigi M, Benigni A, Remuzzi G: In vivo maturation of functional renal organoids formed from embryonic cell suspensions. J Am Soc Nephrol 2012, 23: 1857-1868

10. Francipane MG, Lagasse E: The lymph node as a new site for kidney organogenesis. Stem Cells Transl Med 2015, 4:295-307

11. Francipane MG, Han B, Oxburgh L, Sims-Lucas S, Li Z, Lagasse E: Kidney-in-a-lymph node: a novel organogenesis assay to model human renal development and test nephron progenitor cell fates. J Tissue Eng Regen Med 2019, 13:1724-1731

12. Matsumoto K, Yokoo T, Yokote S, Utsunomiya Y, Ohashi T, Hosoya T: Functional development of a transplanted embryonic kidney: effect of transplantation site. J Nephrol 2012, 25:50-55

13. Francipane MG, Han B, Lagasse E: Host lymphotoxin-beta receptor signaling is crucial for angiogenesis of metanephric tissue transplanted into lymphoid sites. Am J Pathol 2019, 189:2520-2537

14. Francipane MG, Lagasse E: Maturation of embryonic tissues in a lymph node: a new approach for bioengineering complex organs. Organogenesis 2014, 10:323-331

15. Noort AR, van Zoest KPM, Weijers EM, Koolwijk P, Maracle CX, Novack DV, Siemerink MJ, Schlingemann RO, Tak PP, Tas SW: NF$\mathrm{KB}$-inducing kinase is a key regulator of inflammation-induced and tumour-associated angiogenesis. J Pathol 2014, 234:375-385

16. Dejardin E, Droin NM, Delhase M, Haas E, Cao Y, Makris C, Li ZW, Karin M, Ware CF, Green DR: The lymphotoxin-beta receptor induces different patterns of gene expression via two NF-KappaB pathways. Immunity 2002, 17:525-535

17. Malhotra D, Fletcher AL, Astarita J, Lukacs-Kornek V, Tayalia P, Gonzalez SF, Elpek KG, Chang SK, Knoblich K, Hemler ME, 
Brenner MB, Carroll MC, Mooney DJ, Turley SJ: Transcriptional profiling of stroma from inflamed and resting lymph nodes defines immunological hallmarks. Nat Immunol 2012, 13:499-510

18. Malhotra D, Fletcher AL, Turley SJ: stromal and hematopoietic cells in secondary lymphoid organs: partners in immunity. Immunol Rev 2013, 251:160-176

19. Chyou S, Ekland EH, Carpenter AC, Tzeng TCJ, Tian S, Michaud M, Madri JA, Lu TT: Fibroblast-type reticular stromal cells regulate the lymph node vasculature. J Immunol 2008, 181:3887-3896
20. Seleznik G, Seeger H, Bauer J, Fu K, Czerkowicz J, Papandile A, Poreci U, Rabah D, Ranger A, Cohen CD, Lindenmeyer M, Chen J, Edenhofer I, Anders HJ, Lech M, Wüthrich RP, Ruddle NH, Moeller MJ, Kozakowski N, Regele H, Browning JL, Heikenwalder M, Segerer S: The lymphotoxin $\beta$ receptor is a potential therapeutic target in renal inflammation. Kidney Int 2016, 89: $113-126$

21. Xinaris C: Organoids for replacement therapy: expectations, limitations and reality. Curr Opin Organ Transplant 2019, 24:555-561 